\title{
A New Preference Based Model for Relevant Dimension Identification in Contextual Mobile Search
}

\author{
Sondess Missaoui ${ }^{1(凶)}$ and Rim Faiz ${ }^{2}$ \\ 1 LARODEC, ISG, University of Tunis, Le Bardo, Tunisia \\ sondes.missaoui@yahoo.fr \\ 2 IHEC, University of Carthage, Carthage Presidency, Tunis, Tunisia \\ Rim.Faiz@ihec.rnu.tn
}

\begin{abstract}
Mobile search is a significant task in information retrieval and when coupled with context awareness technologies they can become key tools for mobile users for Web search applications. Context awareness techniques can increase the usability of mobile search providing personalized and more focussed content. However, Contextualized Mobile Information Retrieval still remains a challenging problem. This problem is to identify contextual dimensions that improve search effectiveness and should therefore be in the user's focus. We propose a context filtering process based on a new Preference Language Model, and a new relevance measurement. The experiments have been performed with over than 6000 contextual dimensions. The results show the potential of our Preference model in limiting the negative effects of contextual information overload by using the relevance measurement.
\end{abstract}

Keywords: Mobile information retrieval $\cdot$ Context awareness $\cdot$ User's preferences

\section{Introduction}

Web search personalization is the process of customizing search results to the needs of specific users, taking advantage of the knowledge acquired from the analysis of the users navigational behavior and many other informations such as user profile data and users physical context. For mobile applications, Web Search personalization becomes a necessity for many reasons. First, mobile users use mobile devices as one of the most important communication tools for daily life. Thus, they expect that when they need information about something, they will get it right away and quickly. Second, mobile phones have gone from being a simple voice-service to become a multipurpose service platform that makes the collect of data about user's behavior, preferences, physical context and social relations easier and faster. This emergence in the use of Mobiles has established new challenges. In fact, mobile queries are often short and ambiguous that traditional search engines cannot guess the user's information demands accurately.

(C) Springer International Publishing Switzerland 2014

G. Noubir and M. Raynal (Eds.): NETYS 2014, LNCS 8593, pp. 215-229, 2014.

DOI: $10.1007 / 978-3-319-09581-3 \_15$ 
In addition, a user's information need may undergo a change through search situations (context of the mobile user). For example, the same request "Broadway" may used to mean restaurant at one particular situation and may used to mean an American street in Manhattan city in other situations. Therefore, without the knowledge of specific search situation, it would be difficult to recognize the correct sense behind a query. The major deficiency of the existing search engines is that they generally lack context modeling. To this end, an optimal Mobile Search engine must incorporate context information to provide an important basis for identifying and understanding users information needs. For such reason, an interesting aspect emerging in Mobile Information Retrieval (Mobile IR) appeared recently, that is related to the several contextual dimensions that can be considered as new features to enhance the user's request and solve "the mismatch query problem" [14]. Hence, in the mobile information environment, the context is a strong trigger for information needs. So the question is "What contextual dimensions reflect better the information need and lead to the appropriate search results?" Owing by this question, the challenge is about selecting the best contextual information that may help to personalize web search and meeting the users demands.

In this paper, we focus our research efforts on an area that has received less attention which is the context filtering. We have brought a new approach that has addressed two main problems: how to identify the user's context dependency of mobile queries? And how to filter this user's context and select the most relevant contextual dimensions? This article is organized as follows. In Sect. 2, we give an overview of Context awareness for Mobile Search. In Sect.3, we describe our context model. Then in Sect.4, we present our Filtering approach. Then, in Sect.5, we discuss experiments and obtained results. Finally, by Sect.6, we conclude this paper and outline future work.

\section{Related Works on Contextual Mobile Search}

Mobile context presents challenges of changing location and social context, restricted time for information access, and the need to adapt resources with concurrent physical characteristics of mobile devices. Understanding mobile information needs and associated interaction challenges, is fundamental to improve search browsers. Therefore, Mobile IR systems have evolved to take into account both the user's physical environment and situation. Thence, it was so crucial to integrate context-aware techniques to the aim of modeling contextualized mobile search browsers. The work on context-aware approaches focuses on the adaptation of Mobile IR systems to users needs and tasks. These approaches modelize the user's current context, and exploit it in the retrieval process. Various studies such as [30] and [12] are conducted in order to understand the nature of the information needs of mobile users and their research practices. Indeed, in the context of mobile search, users have less time and screen space for browsing long lists of results. According to [30], users describe their needs in shorter query and using less number of queries by session. Also, they usually consult only the 
first page of results. Taking this into account, some recent papers have investigated several techniques of context awareness in Mobile IR to recognize user's intention behind the query such as Bouidghaghen et al. [2], and especially to personalize web search such as Tsai et al. [9], Pitkow et al. [28] and Ahn et al. [27]. Personalized web search aims at enhancing the user's query with the his context to better meet the individual needs. To this aim, many research efforts such as $[1,4,6,24,29]$ are performed to modelize context, allowing to identify information that can be usefully exploited to improve search results. The context can include a wide range of dimensions necessary to that characterize the situation of the user. Also, the situation and information demand have more dynamic change in a mobile environment. Owing to the above characteristics, a big challenge appeared which is about selecting the best contextual information that may help to personalize web search and meeting the users demands.

The Related work in the domain can be summarized in terms of three categories. Firstly, approaches which are characterized as "one dimension fits all" using one same contextual dimension to personalize all search queries. Secondly, approaches such as [20] that exploit a set of predefined dimensions for all queries even though these latter are submitted by different users in different contexts. And finally, approaches that are performed to the aim of filtering the user's context and exploit only the relevant information to personalize the mobile search: in this category, our work has proceeded in terms of filtering the mobile context and identifying relevant dimensions to be latter using in contextual ranking approach. The one dimension fits all approaches consider user's mobile context as one dimension at a time sessions. In this category, several research efforts are proposed in the literature to modelize the current user's situation, where location is probably the most commonly used variable in context recognition. Some of these approches such as Bouidghaghen et al. [2], Welch and Cho [21], Chirita et al. [4], Vadrevu et al. [23] and Gravano et al. [18] have build models able to categorize queries according to their geographic intent. Based on localization as the most relevant factor, they define query as "Global" or "local implicit". Indeed, they identify the query sensitivity to location in order to determine whether the user's need is related to his geographic location (local implicit) or not (Global). For local search queries, those approaches personalize the search results using current location. In the same category, Welch and Cho [21], Vadrevu et al. [23] and Gravano et al. [18] use the classification techniques to achieve this goal. Bouidghaghen et al. [2] propose a location query profile in order to automatically classify location sensitive queries on "Local implicit, Local explicit and Global". Location can be considered as an important context dimension but in this field it is not the only one, others can be taken into account. Some queries have no intent for localization (e.g. Microsoft office version, Horoscope) but they are "Time" sensitive. With the aim of recognizing user's intention behind the search, using a unique predefining context's dimension is not accurate. For example, when a mobile user is a passenger at the airport and he is late for check-in, the relevant information often depends in more than time or localization. It is a complex searching task. So, it needs some additional context dimensions such 
as activities and dates (e.g., flight number inferred from the user's personal calendar or numeric agenda). As another example let us consider a group of users are preparing for an outing with friends for the weekend. If the query "musical event" is formulated by one of them, the query evaluation should produce a different contextual dimensions such as location, time and preferences. The second category of approaches propose to use a set of contextual dimensions for all queries and do not offer any context adaptation models to the specific goals of the users. Several works in this category use 'Here' and 'now', both as the main important concepts for mobile search. Thus, projects as MoBe project of Coppola et al. [5] and Castelli et al. [3] operate including Time and Location as main dimensions besides others in which Bayesian networks are used to automatically infer the user's current context. Most of these approaches use generally classification techniques, and few studies have tried to use semantics and ontological modeling techniques for context such as Gross and Klemke [19], Jarke et al. [10] and Aréchiga et al. [1]. In Mymose system, Aréchiga et al. [1] propose a multidimensional context model, which includes four main dimensions (Spatial, Temporal, Task and Personal model). Those dimensions are supported by ontologies and thesaurus to represent the knowledge required for the system. While all aspects of the operational mobile environment have the potential to influence the outcome search results, only a subset is actually relevant. For such reason, the last category of approaches such as [11,17] are proposed to identify the appropriate contextual information in order to tailor the search engine results to the specific user's context. Kessler [11] approach is built to automatically identifying relevant context parameters. He proposes a cognitively plausible dissimilarity measure "DIR". The approach is based on the comparison of result rankings stemming from the same query posed in different contexts. This measure aims to calculate the effects of context changes on the IR results. Another research effort, Stefanidis et al. [17], specify context as a set of multidimensional attributes. They identify user's preferences in terms of their ability to tailor with the context state of a query. In order to improve understanding the user's needs and to satisfy them by providing relevant responses, we propose a novel model inspired by the last category of approaches. It allows to define the most relevant and influential user's context dimensions for each search situation. Comparing to the previously discussed approaches, our main contribution is to filter the mobile user's context in order to tailor search results with the intention behind his query. We formulate the context filtering problem as the problem of identifying those contextual dimensions whic are eligible to encompass the user's preferences. We provide a new score that allows to compute the relevance degree of each dimension. The idea is: "the more relevant the context dimension is, the more effective the personalized answer can be".

\section{Our User's Context Model}

Within Mobile IR research, context information provides an important basis for identifying and understanding users information needs [7]. In fact, mobile devices 


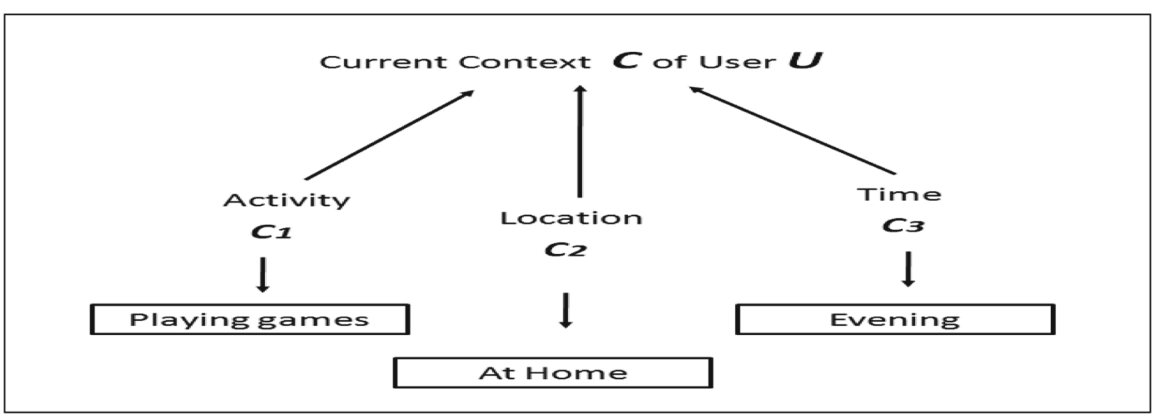

Fig. 1. An instance of a modeled context.

provide more context information than personal computers, such as location information, Time, SMS Clicks, Friends Contacts, Tags, Profiles, Traffic, and certain social networks. All those can be considered as context dimensions and then be used as additional features to create an efficient representation of the user's situation. In our work, the context is modeled through a finite set of special purpose attributes, called context dimensions $c_{i}$, where $c_{i} \in C$ and $C$ is a set of $\mathrm{n}$ dimensions $\left\{c_{1}, c_{2} \ldots, c_{n}\right\}$ For instance we adopt a context model that represents the user's context by only three dimensions Location, Time, Activity. The Fig. 1 shows the concept of user's context $C$ with an instance of a finite set of contextual dimensions Location, Time, Activity. The user's current context is the user's physical, environmental and organizational situations in the moment of search. It can be considred as the current state at the time of the query submission. For example, when a query such as "Restaurant" is formulated by a parent, his current situation can be definite as Location: Sousse - Tunisia; Time: Evening-12/09/2012; Activity: Outing with family. We present in the next section our filtering model including the main features that allow to filter the user's current context and specify the most relevant contextual dimensions to narrow the search.

\section{Context Filtering Process Based on Preference Language Model}

A user's context is multidimensional and contains lots of information. Those dimensions are changing from one situation to another and may have an important effect for a query and haven't the same importance for another. Hence, including all context's dimensions for each search can decrease the reliability and robustness of search engine in terms of response time and efficiency. Thus, we propose to filter the context in order to retain only relevant dimensions. In order to identify relevant contextual dimension we measure the effect of each dimension on mobile queries performance according to two language models: 
- Query Language Model proposed by Cronen et al. [22]

- and our Preference Language Model, which we proposed and described in this section.

\subsection{Query Language Model}

Query Language Model is used in many research such as [8] and [2] to the aim of analyzing the user's query and its sensibility to the context. However the best way to analyze a context dimension is to look at its effect on the query. Thence, its effect on the type of documents the query retrieves. In our work we use the Query language model approach as described in [22] which allow to examine the performance of the query. To this aim, we follow those steps:

- Step 1: We begin by selecting the top N (cf. Sect.5) search results of intial user's query $\left(Q_{i n}\right)$.

- Step 2: In the second step, we refine the query by adding the contextual dimension $c_{i}$. We obtaine a refined query $\left(Q_{c_{i}}\right)$ for which we select also the top $\mathrm{N}$ search results.

- Step 3: We measure the effect of the dimension $c_{i}$ on the query outcomes by comparing the search results of both intial query $\left(Q_{i n}\right)$ and refining query $\left(Q_{c_{i}}\right)$ using the language model as described in [22]. The assumption is that the more the dimension enhance this model the more it is relevant.

In a language modeling approach $[13,16]$, we rank the documents in a collection according to their likelihood of having generated the query. Given a query $Q$ and a document $\mathrm{D}$,this likelihood is presented by the following equation:

$$
P(Q \mid D)=\prod_{w \in Q} P(w \mid D)^{q_{w}}
$$

We denote, $q_{w}$ as the number of times the word $w$ occurs in query $Q$ which was restricted to 0 or 1 . Thus, in [25] the document language models $P(w \mid D)$, are estimated using the words in the document. This ranking allows to build a query language model, $P(w \mid Q)$, out of the top N documents:

$$
P(w \mid Q)=\sum_{D \in R} P(w \mid D) \frac{P(Q \mid D)}{\sum_{D \in R} P\left(Q \mid D^{\prime}\right)}
$$

Where $\mathrm{R}$ is the set of top $\mathrm{N}$ documents. At this point, we use Kullback-Leibler divergence to calculate the gap between the query language models (unigram distributions) of $\left(Q_{i n}\right)$ and $\left(Q_{c_{i}}\right) .\left(Q_{i n}\right)$ is the initial query submitted by the user. $\left(Q_{c_{i}}\right)$ is the refined query by adding the contextual dimension $c_{i}$ to $\left(Q_{i n}\right)$. Thus, This gap between the both models, can be considered as the effect of the contextual dimension $c_{i}$ (cf. Sect.5.1) on the mobile query performance.

$$
D_{k l}\left(P\left(w \mid Q_{c_{i}}\right), P\left(w \mid Q_{i n}\right)\right)=\sum_{w \in Q} P\left(w \mid Q_{c_{i}}\right) \log \frac{P\left(w \mid Q_{c_{i}}\right)}{P\left(w \mid Q_{i n}\right)}
$$

Where $P\left(w \mid Q_{i n}\right)$, is the language model of the initial query, used as a background distribution. $P\left(w \mid Q_{c_{i}}\right)$ is the language model of the refined query. 


\subsection{Preference Language Model}

In the following, we propose a preference language model to incorporate user's preferences information into the language model so that we induce the effictivness of a query to return results related to user's preferences. Which mean, its ability to describe well the user's information need. Thus we wish to examine some preferences profile of a query Q. E.g., Searching for some "events", the mobile search system must take into account the user's preference "Art". Hence relevant retrieved results must contain cultural or musical events. By analogy to the "Query Language Model", we create a "Preferences Language Model" described as the maximum likelihood estimates.

$$
\hat{P}(\text { Pre } \mid Q)=\sum_{D \in R} \hat{P}(\text { Pre } \mid D) \frac{P(Q \mid D)}{\sum_{D \in R} P(Q \mid D)}
$$

Where "Pre" is a term that describes a user preferences category from a data base containing all user's preferences (his profile). For example if a user is interested by "Music" a set of terms such as (Classical songs, Opera, Piano, Saxophone) are defined as "Pre". The maximum likelihood estimate of the probability "Pre" under the term distribution for document D is:

$$
\hat{P}(\text { Pre } \mid D)= \begin{cases}1 & \text { if Pre } \in \text { Pre }_{D} \\ 0 & \text { Otherwise }\end{cases}
$$

Where $\operatorname{Pre}_{D}$ is the set of categories names of interests contained in document D (e.g. Art, Music, News, Cinema, Horoscope ... ). The profile, that describes the user's interests and preferences could be explicitly set by the user or gathered implicitly from the user search history. In our experiments, a profile is collected explicitly before starting the search session. Similar to a regular query language model, the preference model also needs to be smoothed to solve the zero probability problem and to penalize common terms. To this aim, We consider a popular smoothing method Jelinek-Mercer [26]. We use the distribution of the initial query $Q_{i n}$ (reference-model) over preferences as a background model. This background model is defined by:

$$
\hat{P}\left(\text { Pre } \backslash Q_{i n}\right)=\frac{1}{|N|} \sum_{D} \hat{P}(\text { Pre } \mid D)
$$

Thus, the smoothing method is given by:

$$
P^{\prime}(\text { Pre } \mid Q)=\lambda \hat{P}(\text { Pre } \mid Q)+(1-\lambda) \hat{P}\left(\text { Pre } \mid Q_{i n}\right)
$$

Given $\lambda$ as a smoothing parameter.

Figure 2 shows an example for the effect of different dimensions on the Preference Language Model (preferences profile) of the query "Olympic sports". For our experiments, we choose as dimensions three contextual informations which are Time, Location and Activity. In this example the initial query presents a flat preferences profile, while Time, location and Activity dimensions have profiles 


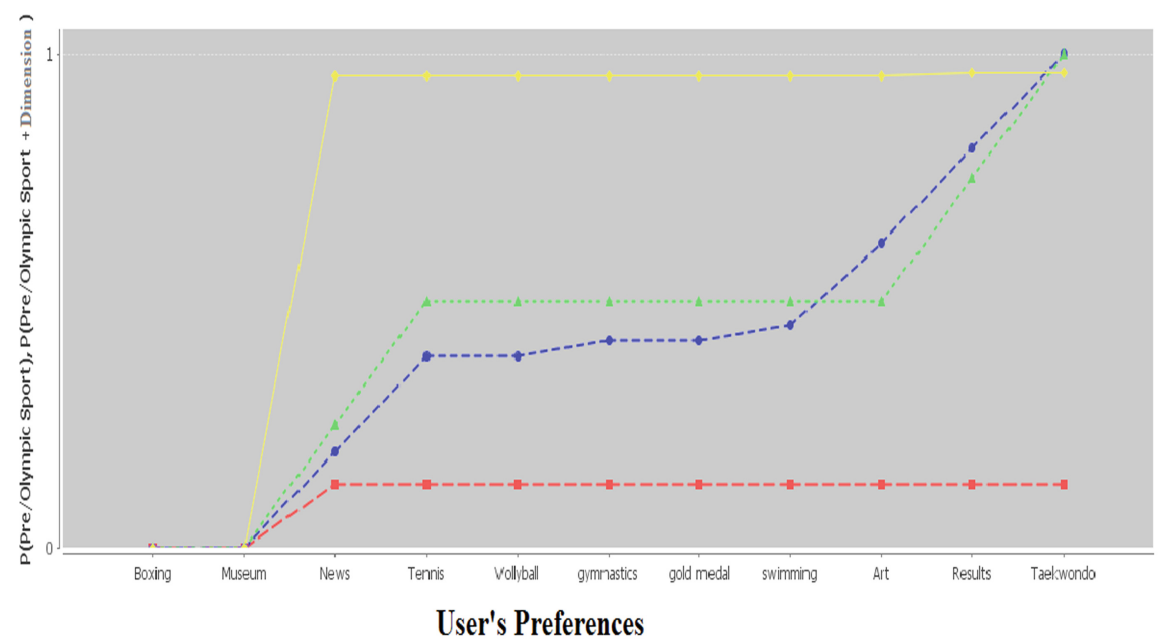

\section{Preference Query Profile + Preference Profile for Query+Location $=$ Preference Profile for Query+Time + Preference Profile for Query+Activity}

Fig. 2. Comparison between the preferences profiles of the query "Olympic sports", an enhanced queries using three contextual dimensions.

with distinctive peaks spread over many user's preferences. Looking in depth on this graph of Fig. 2, we can see the difference between calculated preferences profile for the initial query $\left.P\left(\operatorname{Pre} \mid Q_{i n}\right)\right)$ and preference profile for the query enhanced by a contextual dimension $c_{i}$ is "Time" $P\left(\right.$ Pre $\left.\mid Q_{\text {Time }}\right)$. Indeed, Time dimension makes a clear improvement for some preferences (Results, Taekwando) over the others profiles. But for some others user's preferences $P\left(\right.$ Pre $\left.\mid Q_{\text {Time }}\right)$ is less or equal to $P\left(\operatorname{Pre} \mid Q_{i n}\right)$ ). The assumption of this analysis is that irrelevant dimension's Preference Language Model show no variance comparing to the initial query Preference Language Model. Given that, this contextual dimension is not important for a query and shouldn't be selected. In contrast, a relevant dimension provides query Preference Language Model with at least one peak. At this point, we need to analyze a context dimension's effect on the Preference Language Model. Thence, we follow those steps:

- Step 1: We begin by selecting the top N (cf. Sect. 5) search results of intial user's query $\left(Q_{i n}\right)$. Then we calculate the Preference language model of $Q_{i n}$.

- Step 2: In the second step, we refine the query by adding the contextual dimension $c_{i}$. We obtaine a refined query $\left(Q_{c_{i}}\right)$ for which we select also the top $\mathrm{N}$ search results. Also, Then we calculate the Preference language model of $Q_{c_{i}}$.

- Step 3: We measure the effect of the dimension $c_{i}$ on the Preference language model using Kullback-Leibler divergence. 
Using Kullback-Leibler divergence, we can estimate the effect of the dimension on Preference Language Model, which is initially defined as:

$$
\begin{array}{r}
D_{k l}\left(P\left(\text { Pre } \mid Q_{c_{i}}\right), P\left(\text { Pre } \mid Q_{i n}\right)\right)= \\
\sum_{{\text {Pre } \in \text { Pre }_{D}}_{P} P\left(\text { Pre } \mid Q_{c_{i}}\right) \log \frac{P\left(\text { Pre } \mid Q_{c_{i}}\right)}{P\left(\text { Pre } \mid Q_{i n}\right)}}
\end{array}
$$

Where $P\left(\right.$ Pre $\left.\mid Q_{c_{i}}\right)$ is Preferences Language Model for the refined query $Q_{c_{i}}$ using a contextual dimension $c_{i}$. At this level, we need to calculate the general effect (improvement/non improvement) of the dimension on Preference Language Model as on Query Language Model to predict its relevance degree.

\subsection{Identification of Relevant Dimensions Using Relevance Measurement}

We introduce a new measure that allows to specify the relevance of a contextual dimension. It defines the performance of the dimension at enhancing the mobile query. Our measure named "Relevance score" combines linearly the effect of such dimension on Query Language Model and Preference Language Model using the following formula:

$$
\begin{gathered}
\text { Relevance }\left(c_{i}, Q\right)= \\
{\left[D_{k l}\left(P\left(\text { Pre } \mid Q_{c_{i}}\right), P\left(\text { Pre } \mid Q_{i n}\right)\right)+D_{k l}\left(P\left(w \mid Q_{c_{i}}\right), P\left(w \mid Q_{i n}\right)\right)\right]}
\end{gathered}
$$

with Relevance $\left(c_{i}, Q\right)$ on $[0,1]$. where $c_{i}$ and $C$ represent respectively, contextual dimension and user's current context. Once this Relevance score is calculated, we define experimentally a threshold value $\gamma$. A relevant dimension $c_{i}$ must have a relevance degree that goes beyond $\gamma$, otherwise it is considered irrelevant and will be not including in the personalization as an element of the accurate user's current context $C$. The proposed context-based measurement model can be expressed in a formal manner with the use of basic elements toward mathematic interpretation that build representative values from 0 to 1 , corresponding to the intensity of dimension's relevance. Being null values indicative of non importance for that dimension (it should not be integrated in personalization of mobile information retrieval process). In the experiment, we will try to define the threshold that a dimension should obtain to be classified as relevant or irrelevant information. In the next section, we will also evaluate the effectiveness of our metric measure "Relevance score" to classify the contextual dimensions.

\section{Experimental Evaluation}

Our goal is to evaluate the "Relevance score" measure. In this section, we present our training and test collection, our evaluation protocol, which has been greatly improved compared to [15], then we describe and discuss the obtained results. We have improved our system performance which is achieved, through the size augmentation of our database (from 300 to 2200 queries), and the creation of a new user's context model with over than 6000 dimensions. which enhances the stability and reliability of our system. 


\subsection{Dataset}

For the experiments reported in this work, we used a real-world dataset which is a fragment submitted to the America Online search engine. We had access to a portion of the 2006 query $\log$ of $\mathrm{AOL}^{1}$. We had relied on some experts in the field of Information Retrieval to pick manually 2200 initial set of queries based on the signification of their terms. Also experts have select queries which may be related to the user's environmental and physical context. After a filtering step to eliminate duplicate queries, we obtained a set of 2000 queries. Where three contextual dimensions (Time, Location and Activity) are assigned to each query to indicate the user current situation. To obtain the top N Web pages that match each query, we use a Web search engine namely Google via the Google Custom Search $\mathrm{API}^{2}$. We considered only the first 10 retrieved results, which is reasonable for a mobile browser, because mobile users aren't likely to scroll through long lists of retrieved results. Then, for each query in the test set we classified manually their related contextual dimensions. Each dimension is associated to a label to indicate whether it is irrelevant or relevant. The criterion to assess whether a given dimension is relevant, is based on whether the mobile user expects to see search results related to this contextual information ordered high on the results list of a search engine. E.g., for a query such as "weather" the user can express his intention to see search results related higher to location and time information. Since, these dimensions are judged relevant. These steps left us, in our sample test queries, with $34 \%$ irrelevant dimensions and $65.6 \%$ relevant.

\subsection{Evaluation Protocol: Assessment of the Classification Performance of Relevance Score}

Our experimental design allows us to evaluate the effectiveness of our technique to identify user's relevant contextual dimensions. For this purpose, we propose an evaluation methodology of obtained results using manually labeled contextual dimensions. In fact, a contextual dimension's class is correct only if it matches the labeled results. Using the Relevance score as a classification feature, we build a context intent classifier. In order to compute the performance of the classifiers in predicting the parameter types, we use standard precision, recall and F-measure measures. We use also classifiers implemented as part of the Weka ${ }^{3}$ software. We test the effectiveness of several supervised individual classifiers (Decision trees, Naive Bayes, SVM,and a Rule-Based Classifier) in classifying contextual parameters using Relevance score as classification feature.

\footnotetext{
${ }^{1}$ http://www.gregsadetsky.com/aol-data/

${ }^{2}$ https://developers.google.com/custom-search/

${ }^{3}$ http://www.cs.waikato.ac.nz/ml/weka/
} 


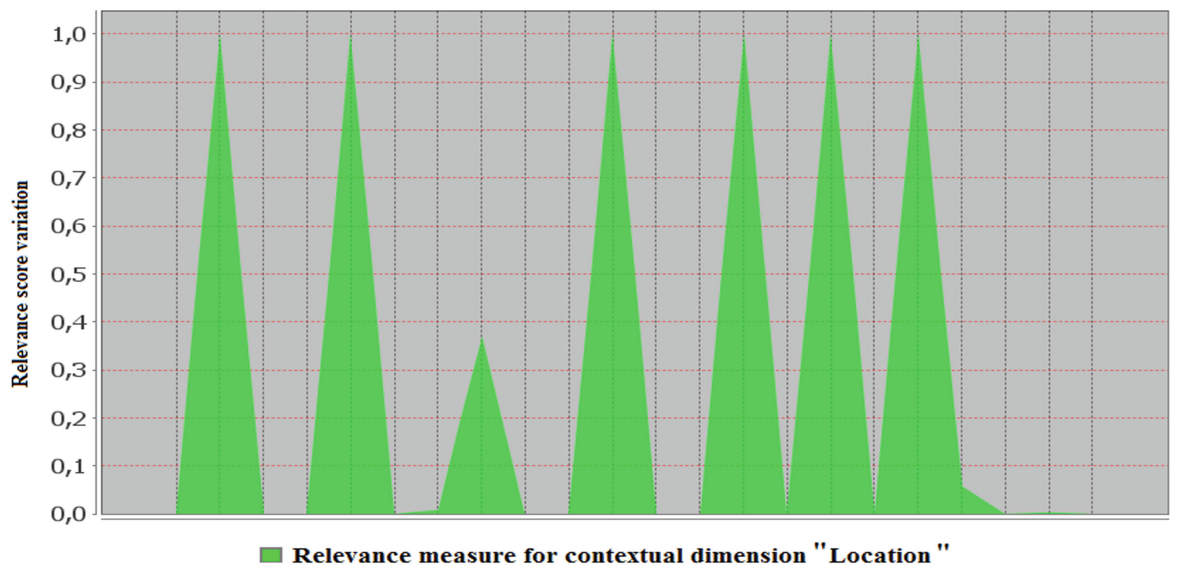

Fig. 3. Distribution of Relevance measure for geographic dimension (Location).

\subsection{Results and Discussion}

Analysis of Relevance Score Measure. At this level we analyze the "Relevance score" distribution for each category of contextual parameters. Figure 3 shows distribution of Relevance score measure over different values of Location parameters for different queries. In this figure we notice that there is a remarkable drops and peaks in the value of 'Relevance score' for the Location parameters. Moreover, the distribution of this measure for temporal parameters, presented in Fig. 4, has a clear variation with multiple values which clearly support our assumption here. Indeed, the relevance of a contextual parameter is independent on his type or value but it depends on the query and the intention of mobile user behind such query. Hence, Relevance score measure hasn't a uniform distribution for those contextual parameters. It is still depending on the user's query. We can conclude that the measure based on language model approach succeeds to measure the sensitivity of user's query to each contextual dimension.

Table 1 presents the two lowest and two highest values for each parameter class, obtained from our sample test queries and their user's context. Those values allow to confirm a possible correlation between parameter intent class and 'Relevance score' feature. Hence, we define a threshold value for each parameter class. And in the following, we will evaluate the effectiveness of thus thresholds to classify those dimensions.

Effectiveness of Contextual Parameter Classification. Our goal in this evaluation is to assess the effectiveness of our classification attribute 'Relevance score' to identify the type of contextual dimension from classes: relevant and irrelevant. As discussed above, we tested different types of classifiers and Table 2 presents the values of the evaluation metrics obtained by each classifier. In fact, all the classifiers were able to distinguish between the both contextual parameter 


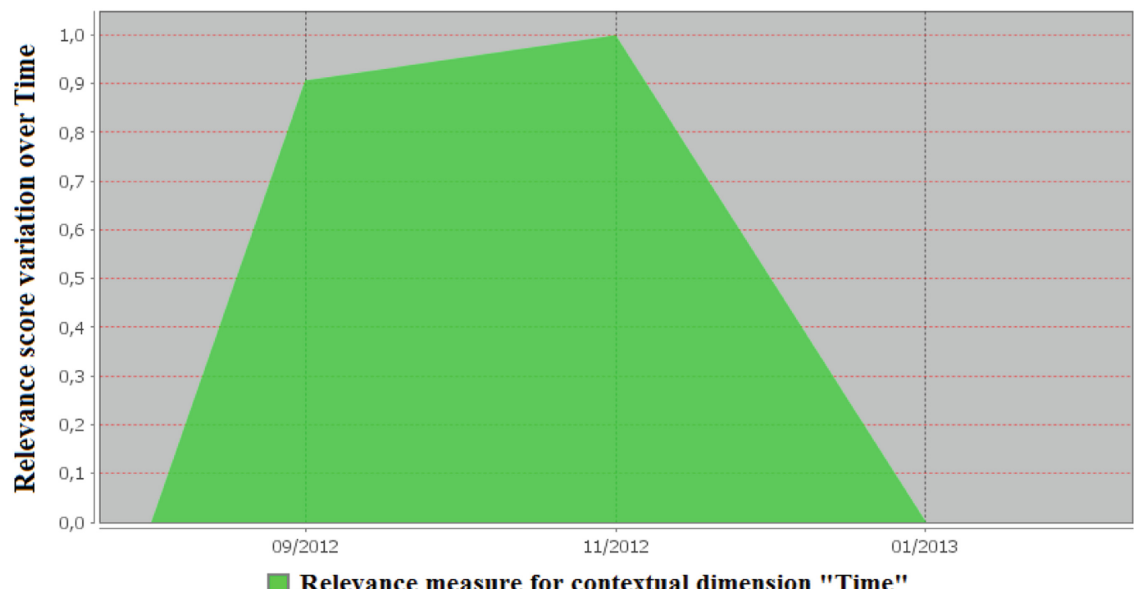

Fig. 4. Distribution of Relevance measure for temporal dimension (Time).

Table 1. The fourth lowest and highest values for each dimension class.

\begin{tabular}{l|l|l|l}
\hline Query & Parameters & Relevance score & Class \\
\hline "house for sale" & Activity: At home & 0.432 & Relevant \\
\hline "check in airport" & Time: $12 / 09 / 2012$ & 0.550 & \\
\hline "house for sale" & Location: Tunisia & 0.985 & \\
\hline "check in airport" & Activity: Walking & 0.999 & \\
\hline "outdoor tiki hut bars" & Time: 30/08/2012 & 0.147 & \multirow{2}{*}{ Irrelevant } \\
\hline "eye chart" & Activity: Working & 0.169 & \\
\hline "weather" & Activity: Walking & 0.199 & \\
\hline
\end{tabular}

classes. Fmeasures, Precision and Recall ranging from $96 \%$ to $99 \%$. But "SVM" classifier achieves the highest accuracy with $99 \%$ for the F-measure. This first experiment implies the effectiveness of our approach to accurately distinguish the both types of user's current contextual information. It especially allows to correctly identify irrelevant contextual information with an evaluation measure over 1 . When relevant achieving over $97 \%$ classification accuracy.

In a second experiment, we evaluated the classification effectiveness of our approach comparatively to DIR approach developed by Kessler [11]. The DIR measure enables distinguishing between irrelevant and intended context. Whence, we compared the two approaches only on this basis. We implemented the DIR approach using the SVM classifier which achieves one of the best classification performance using one simple rule: analyzing the individual results in two rankings for the same query expanded by different contextual parameters. Intended or relevant contextual information must have an impact that goes beyond a threshold value. 
Table 2. Classification performance obtained using a classifier with Relevance score as a feature.

\begin{tabular}{|c|c|c|c|c|c|}
\hline Classifier & Class & Precision & Recall & F-measure & Accuracy \\
\hline \multirow[t]{3}{*}{ SVM } & Relevant & 0.978 & 0.989 & 0.981 & \multirow[t]{3}{*}{$99 \%$} \\
\hline & Irrelevant & 1 & 1 & 1 & \\
\hline & Average & 0.991 & 0.99 & 0.99 & \\
\hline \multirow[t]{3}{*}{ JRIP rules } & Relevant & 0.911 & 0.953 & 0.924 & \multirow[t]{3}{*}{$96.3 \%$} \\
\hline & Irrelevant & 1 & 1 & 1 & \\
\hline & Average & 0.965 & 0.962 & 0.962 & \\
\hline \multirow[t]{3}{*}{ Bayes } & Relevant & 1 & 0.933 & 0.966 & \multirow[t]{3}{*}{$97 \%$} \\
\hline & Irrelevant & 1 & 1 & 1 & \\
\hline & Average & 0.973 & 0.971 & 0.971 & \\
\hline \multirow[t]{3}{*}{$\mathrm{J} 48$} & relevant & 1 & 0.933 & 0.966 & \multirow[t]{3}{*}{$97 \%$} \\
\hline & irrelevant & 1 & 1 & 1 & \\
\hline & average & 0.973 & 0.971 & 0.971 & \\
\hline
\end{tabular}

Table 3. Classification performance on Relevant and Irrelevant dimensions: comparison between our approach and DIR measure approach.

\begin{tabular}{l|l|l|l|l|l|l|l|l|l}
\hline Approach & DIR approach & \multicolumn{2}{l}{ Our approach: Relevance score } \\
\hline Class & Relevant & Irrelevant & Average & Relevant & Impro & Irrelevant & Impro & Avrege & Impro \\
\hline Precision & 1 & 0.968 & $\mathbf{0 . 9 8 2}$ & 1 & $\mathbf{0 \%}$ & 0.984 & $\mathbf{1 . 7} \%$ & $\mathbf{0 . 9 9 1}$ & $\mathbf{1 \%}$ \\
\hline Recall & 0.956 & 1 & $\mathbf{0 . 9 8 1}$ & 0.978 & $\mathbf{2 . 3} \%$ & 1 & $\mathbf{0 \%}$ & $\mathbf{0 . 9 9}$ & $\mathbf{1 \%}$ \\
\hline F-measure & 0.977 & 0.984 & $\mathbf{0 . 9 8 1}$ & 0.989 & $\mathbf{1 . 3} \%$ & 0.992 & $\mathbf{0 . 9} \%$ & $\mathbf{0 . 9 9}$ & $\mathbf{1 \%}$ \\
\hline Accuracy & $\mathbf{9 8 \%}$ & \multicolumn{7}{|c|}{$\mathbf{9 9 . 5 \%}$} \\
\hline
\end{tabular}

Hence, we should obtain a high value of DIR measure to classify a context as relevant. Table 3 presents the precision, recall, F-measure and accuracy achieved by the SVM classifier according to the both approaches. The result of comparison show that, our approach gives higher classification performance than DIR approach with an improvement of $1 \%$ at accuracy. This improvement is mainly over Relevant context parameters with $1.3 \%$ at Recall.

\section{Conclusion}

We proposed in this paper a filtering model for mobile user's context that evaluates the quality of contextual dimensions using different features and selects the most appropriate among them. Those dimensions will improve the retrieval process to produce reliable and in context results. This approach is based on the language models approach. We have built a new metric measure namely Relevance score that allows to effectively classify the contextual dimensions on Relevant and irrelevant according to their ability to enhance the search results. 
Our experimental evaluation on a part of the AOL corpus shows that some contextual dimensions are more relevant and influential then others. Whence, we evaluated the classification performance of our metric measure comparatively to a cognitively plausible dissimilarity measure namely DIR. For future work, we plan to extend filtering model using others contextual dimensions and to exploit our proposed automatic method to personalize mobile Web search. We will customize the search results for queries by considering the determined user's contextual dimensions classified as relevant.

\section{References}

1. Aréchiga, D., Vegas, J., De la Fuente Redondo, P.: Ontology supported personalized search for mobile devices. In: Proceedings of the ONTOSE (2009) (Mymose)

2. Bouidghaghen, O.: Accés contextuel à l'information dans un environnement mobile: approche basée sur l'utilisation d'un profil situationnel de l'utilisateur et d'un profil de localisation des requêtes. Thesis of Paul Sabatier University (2011)

3. Castelli, G., Mamei, M., Rosi, A.: The whereabouts diary. In: Hightower, J., Schiele, B., Strang, T. (eds.) LoCA 2007. LNCS, vol. 4718, pp. 175-192. Springer, Heidelberg (2007)

4. Chirita, P., Firan, C., Nejdl, W.: Summarizing local context to personalize global Web search. In: Proceedings of the Annual International Conference on Information and Knowledge Management, pp. 287-296 (2006)

5. Coppola, P., Della Mea, V., Di Gaspero, L., Menegon, D., Mischis, D., Mizzaro, S., Scagnetto, I., Vassena, L.: CAB: the context-aware browser. IEEE Intell. Syst. 25(1), 38-47 (2010)

6. De Virgilio, R., Torlone, R.: Modeling heterogeneous context information in adaptive web based applications. In: Proceedings of ICWE 2006, 6th International Conference on Web Engineering, pp. 56-63. IEEE Computer Society (2006)

7. Abowd, G.D., Dey, A.K.: Towards a better understanding of context and contextawareness. In: Gellersen, H.-W. (ed.) HUC 1999. LNCS, vol. 1707, pp. 304-307. Springer, Heidelberg (1999)

8. Diaz, F., Jones, R.: Using temporal profiles of queries for precision prediction. In SIGIR, vol. 4, pp. 18-24, July 2004

9. Tsai, F.S., Xie, X., Lee, W.C., Yang, Q.: Intro for mobile IR mobile information retrieval: a survey. J. Eur. J. Sci. Res. 55, 394-400 (2010)

10. Jarke, M., Klemke, R., Nicki, A.: Broker's lounge-an environment for multidimensional user-adaptive knowledge management. In: Proceedings of the 34th Annual Hawaii International Conference on System Sciences, 3-6 Jan 2001, pp. 1-10 (2001). doi:10.1109/HICSS.2001.926339

11. Kessler, C.: What is the difference? a cognitive dissimilarity measure for information retrieval result sets. J. Knowl. Inf. Syst. 30(2), 319-340 (2012)

12. Kamvar, M., Baluja, S.: A large scale study of wireless search behavior: google mobile search. In: Proceedings of the SIGCHI Conference on Human Factors in Computing Systems, ACM Journal, pp. 701-709 (2006)

13. Lavrenko, V., Croft, W.B.: Relevance-based language models. In: Proceedings of the 24th International ACM SIGIR Conference on Research and Development in Information Retrieval, vol. 24, pp. 120-127 (2001)

14. Mario, A., Cantera, J.M., Fuente, P., Llamas, C., Vegas, J.: Knowledge-Based Thesaurus Recommender System in Mobile Web Search (2010) 
15. Missaoui, S., Faiz, R.: Context filtering process for mobile web search. In: Proceedings of the 10th ACS/IEEE International Conference on Computer System and Application (AICCSA 2013)

16. Ponte, J.M., Croft, W.B.: A language modeling approach to Information retrieval. In: Proceedings of the 21st International ACM SIGIR Conference on Research and Development in Information Retrieval, pp. 275-281 (1998)

17. Stefanidis, K., Pitoura, E., Vassiliadis, P.: Adding context to preferences. In: Proceedings of the 23rd International Conference on Data Engineering (ICDE), vol. $23(2007)$

18. Gravano, L., Hatzivassiloglou, V., Lichtenstein, R.: Categorizing web queries according to geographical locality. In: Proceedings of the Twelfth International Conference on Information and Knowledge Management, pp. 325-333 (2003)

19. Gross, T., Klemke, R.: Context modelling for information retrieval: requirements and approaches. J. WWW/Internet 1, 29-42 (2003)

20. Yau, S., Liu, H., Huang, D., Yao, Y.: Situation-aware personalized Information retrieval for mobile internet. In: Proceedings of the Annual International Computer Software and Applications Conference, vol. 27 (2003)

21. Welch, M., Cho, J.: Automatically identifying localizable queries. In: Proceedings of the 31st Annual International ACM SIGIR Conference on Research and Development in Information Retrieval, vol. 31, pp. 1185-1186 (2008)

22. Cronen-Townsend, S., Zhou, Y., Croft, W.B.: Predicting query performance. In: Proceedings of the 25th Annual International ACM SIGIR Conference on Research and Development in Information Retrieval, pp. 299-306. ACM Press, (2002). Commun. ACM J. 50-55 (2002)

23. Vadrevu, S., Zhang, Y., Tseng, B., Sun, G., Li, X.: Identifying regional sensitive queries in web search. In: Proceedings of the 17th International Conference on World Wide Web WWW '08, vol. 8, pp. 507-514 (2008)

24. Poslad, S., Laamanen, H., Malaka, R., Nick, A., Buckle, P., Zipf, A.: Crumpet, creation of user-friendly mobile services personalised for tourism. In: J. 3G Mobile Communication Technologies, pp. 28-32 (2001)

25. Croft, W.B., Lafferty, J.: Language Modeling for Information Retrieval. Kluwer Academic Publishers, Dordrecht (2003)

26. Jelinek, F., Mercer, R.L.: Interpolated estimation of markov source parameters from sparse data. In: Proceedings of the Workshop on Pattern Recognition in Practice (1980)

27. Ahn, J., Brusilovsky, J.P., He, D., Grady, J., Li, Q.: Personalized web exploration with task modles. In: Proceedings of the International Conference on World Wide Web, vol. 17, pp. 1-10 (2008)

28. Pitkow, J., Schutze, H., Cass, T., Cooley, R., Turnbull, D., Edmonds, A., Adar, E., Breuel, T.: Personalized search. Commun. ACM J. 45(9), 50-55 (2002)

29. Ingwersen, P., Jarvelin, K.: The Turn: Integration of Information Seeking and Retrieval in Context. Springer, Dordrecht (2005)

30. Hollan, J.D., Sohn, T., Li, K.A., Griswold, W.G.: A Diary Study of Mobile Information Needs. In: Proceedings of the Data Collection, Florence, Italy (2008) 